\title{
MALIGNANT MELANOMA OF UTERINE CERVIX IN A YOUNG FEMALE
}

\section{Oncology \\ Dr Purvi Rathod}

Dr Ashok Diwan*

Author

Dr Subeera Khan Senior Resident, Department Of Radiation Oncology, GMCH Nagpur, India.

\section{ABSTRACT}

Malignant melanoma is a rare cancer, its incidence being 1\% of all cancers. Malignant melanoma of the female genital tract is even rarer with incidence of $3 \%$ to $7 \%$ of all malignant melanomas. It usually occurs in postmenopausal women. We present a case of malignant melanoma of the uterine cervix in a multiparous premenopausal 29 year old female. She underwent a modified radical hysterectomy. She was stage III and was treated with external beam radiotherapy to the pelvis followed by brachytherapy. Since then the patient was on regular follow up until she developed lung metastasis. Malignant melanoma of the cervix has very less data in literature. Through this case report we will emphasize on the role of radiotherapy in malignant melanoma of uterine cervix.

\section{KEYWORDS}

malignant melanoma, cervical melanoma, radiotherapy, vaginal brachytherapy.

\section{INTRODUCTION}

Malignant melanoma is a rare cancer, its incidence being $1 \%$ of all cancers. Malignant melanoma of the female genital tract is even rarer with incidence of $3 \%$ to $7 \%$ of all malignant melanomas. Malignant melanoma of cervix usually occurs in postmenopausal women, between 60 to 70 years of age ${ }^{[1]}$. The overall prognosis of this highly aggressive disease is very poor since it is diagnosed at an advanced stage and it spreads hematogenously at early stages ${ }^{[2]}$. The cells arise from the melanocytes in the mucosal epithelium of the urogenital system. Malignant melanoma of the cervix has very less data in literature. Here we present a case of malignant melanoma of the uterine cervix in a young female.

\section{Case report}

A 29 year old multipara female, presented with foul smelling blackish brown per vaginal discharge since 6 months. On per speculum examination there was cauliflower like polypoid growth $3 \times 2 \mathrm{cms}$ with blackish brown discharge. On per vaginal examination the growth bled on touch and was arising from the cervix with involvement of anterior fornix. On per rectal examination the rectal mucosa and the bilateral parametrial tissue was free. An excisional biopsy of the cervical mass was done with histopathology s/o malignant cells with melanin deposits favoring malignant melanoma. A careful evaluation was done to rule out other cutaneous sites of malignant melanoma. MRI pelvis revealed soft tissue lesion in the uterine cervix and anterior wall of vagina $3.5 \times 2.7 \times 2 \mathrm{cms}$, there was no parametrial invasion. The lesion was maintaining fat planes with the urinary bladder and the rectum. She underwent a modified radical hysterectomy with bilateral internal iliac and right external iliac lymph node dissection [figure 1]. Her postoperative histopathology was s/o a black growth in the anterior lip of cervix of size $3 \times 2 \mathrm{cms}$ showing melanocytic proliferation in the squamous epithelium. $3 \mathrm{~mm}$ deep infiltration in stroma without vascular invasion ${ }^{\text {[figure } 2]}$. 1 left internal iliac lymph node positive for metastases.

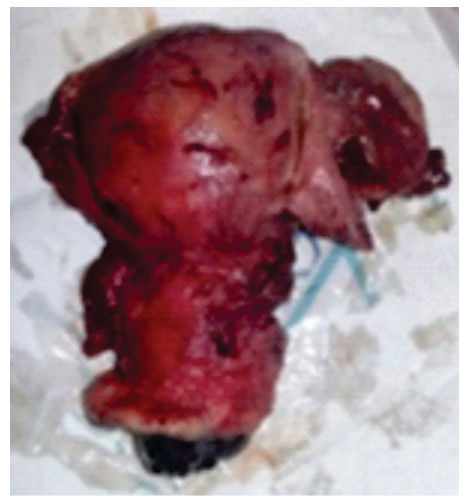

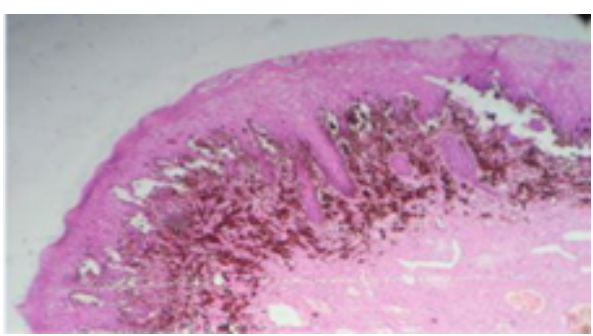

Figure 2

Patient had a past h/o tuberculosis and had undergone left pneumonectomy for bullous changes in the left lung. A metastatic work up with CECT thorax and abdomen was done to rule out distant metastasis. She was stage III (T1N1). The immunohistochemistry revealed the tumor to be positive for HMB-45, MELAN-A, S-100 and Pan cytokeratin. She was treated with External Beam Radiotherapy, dose of 45Gy in 20 fractions was prescribed to the pelvis covering the preoperative tumor volume, parametrium and pelvic nodes. Since vagina was not involved inguinal lymph nodes were not included in radiotherapy fields. the upper border of field was kept at L4-L5 interspace, lower border at obturator foramen and lateral borders at $1.5 \mathrm{cms}$ from the pelvic brim ${ }^{\text {[figure } 3]}$. field size was $17 \times 15 \mathrm{cms}$. antero posterior parallel opposed fields were used. Post EBRT she was treated with intravaginal brachytherapy (HDR) 21Gy in 3 fractions, 7 Gy per fraction dose prescribed at $0.5 \mathrm{~cm}$ depth from the surface of intravaginal cylinder of $3.5 \mathrm{cms}$ diameter. The intention was to cover the vault and upper $2 / 3 \mathrm{rd}$ of vaginal length that was $5 \mathrm{cms}$ in this patient, dose distribution shown in figure 4 [figure 4]. She tolerated radiotherapy well with just grade 2 dermatitis on the gluteal clefts. Post radiation patient was on regular follow up, CECT thorax revealed lung metastases [figure 5]. Patient had a disease free survival (DFS) of 9 months.

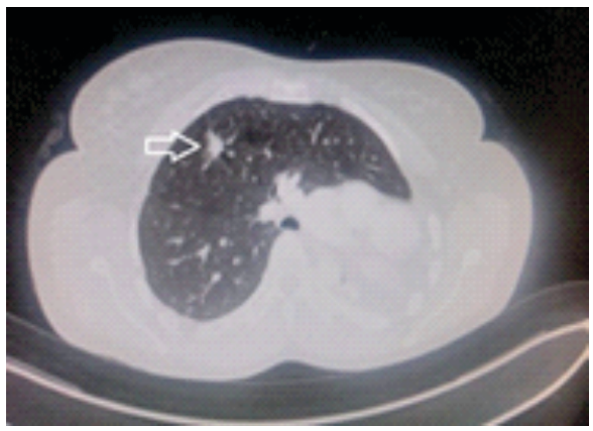

Figure 5
Figure 1

Submitted : $24^{\text {th }}$ May,2019 $\quad$ Revised : $13^{\text {th }}$ July,2019 
She was started on palliative chemotherapy, VDC regimen ( vincristine, dacarbazine, cisplatin) 4 weekly cycles. she tolerated 3 cycles of chemotherapy with grade II diarrhea and 1 episode of febrile neutropenia. however her disease progressed and she expired post $3 \mathrm{rd}$ cycle. her overall survival (OS) was 18 months.

\section{DISCUSSION}

Primary malignant melanomas of the uterine cervix is extremely rare and very aggressive. Cervix is the worst site for malignant melanomas to grow because of limited blood supply and fibrous stroma. Incidence of malignant melanoma of cervix is five times less than that in vagina and vulva. The possibility of cervical melanoma is rare because the organ is not believed to contain any melanocytes, therefore it is mandatory to rule out a primary melanoma elsewhere in the body before confirming the diagnosis as primary cervical malignant melanoma. This highly aggressive disease has tendency for distant metastasis hence a critical workup must be done to exclude distant metastases ${ }^{[3]}$. This includes analysis of serum lactate dehydrogenase, and CT scan of the brain, chest, abdomen and pelvis or combined computed positron emission tomography/tomography scanning of chest, abdomen and pelvis (PET-CT) ${ }^{[4]}$. A complete clinical examination of the skin and oral cavity should be done to rule out cutaneous melanoma. MRI can distinguish a melanoma and other tumors due to high signal pattern from melanin ${ }^{[5]}$. A biopsy is usually sufficient for pathological diagnosis as the histopathology shows melanocytes infiltrating the stroma of the cervical tissue. However if melanin granules are absent and MASSON FONTANA stains negative on histopathology then immunohistochemistry is a must. On IHC S100, HMB-45 show strong positivity.

There is little data available for the definite treatment of cervical malignant melanoma due to its rarity. Surgery is the first therapeutic approach with total hysterectomy with or without bilateral salpingooophorectomy with pelvic lymphadenectomy. The surgical removal of the clinically negative regional lymph nodes is still a controversy. Adjuvant pelvic radiotherapy is considered where the surgical resection is unsatisfactory, parametrial tissue is involved and positive pelvic lymph nodes metastases ${ }^{[6]}$. The role of adjuvant chemotherapy is a matter of debate as no regimen has been able to substantial reduce the chances of recurrence. As far as advanced disease is concerned the role of neoadjuvant chemotherapy has not been established. At present dacarbazine is most widely used chemotherapy drug with a response rate in $15 \%$ to $20 \%$ patients ${ }^{[7]}$ Average survival of these patients ranges from 6 months to 14 years. The majority of them die in the first 3 years after diagnosis ${ }^{[8]}$.

Malignant melanoma of the cervix being a rare case has very less data in literature. Vaginal bleeding $(63.6 \%)$, vaginal mass $(15.9 \%)$, and vaginal discharge $(15.9 \%)$ are common symptoms of genital malignant melanoma ${ }^{[9]}$. A PETCT should be preferred for metastatic workup and a MRI pelvis to know the extent of the disease. The FIGO staging system for cervical cancer has been found to correlate well with the prognosis of the disease ${ }^{[10]}$. If surgical management is possible it should be preferred first followed by adjuvant radiotherapy or chemotherapy or immunotherapy depending on the metastatic status of the patient however the prognosis is usually poor.Our patient developed distant metastases without locoregional recurrence, suggesting the need for immediate adjuvant systemic treatment post completion of radiation therapy.

\section{REFERENCES}

1. Piura P. Management of primary melanoma of the femaleurogenital tract. Lancet Oncol 2008;9:973-8

2. Xia L, Han D, Yang W, Li J, Chuang L, Wu X. Primary malignant melanoma of the vagina: a retrospective clinicopathologic study of 44 cases. Int J Gynecol Cancer. 2014;24:149-155.

3. Piura P. Management of primary melanoma of the female urogenital tract. Lancet Oncol 2008;9:973-8.

4. Patrick RJ, Fenske NA, Messina JL. Primary mucosal melanoma. J Am AcadDermatol 2007;56:828-34.

5. Sugiyama VE, Chan JK, Kapp DS. Management of melanomas of the female genital tract. CurrOpinOncol 2008;20:565-9.

6. Vleugels MP, Brolmann HA, van Beek M. Primary melanoma of the cervix uteri, an avisrara? A review of the literature. ActaObstet GynecolScand 1990;69:259-64.

7. Sugiyama VE, Chan JK, Kapp DS. Management of melanomas of the female genital tract. CurrOpinOncol 2008;20:565-9.

8. C. Siozos, A. Bhat, R. Lonsdale, J. J. Nieto, and S. G. Crocker, "Malignant melanoma of the uterine cervix," Journal of Obstetrics and Gynaecology, vol. 25, no. 8, pp. 826-827, 2005 .

9. Cantuaria G, Angioli R, Fernandez-Abril A, Penalver M. Primary malignant melanoma of the uterine cervix: case report and review of the literature. Prim Care Update Ob Gyns. 1998;5:159-160.

10. Morrow CP, DiSaia PJ. Malignant melanoma of the female genitalia: a clinical analysis. ObstetGynecolSurv. 1976;31:233-271. 